\title{
Research on Cognitive Coupling between Digital Media Space and Real Urban Space in the Era of Big Data A Case Study of Chongqing, China
}

Haixuan Zhu, School of Architecture, Harbin Institute of Technology; Key Laboratory of Cold Region Urban and Rural Human Settlement Environment Science and Technology, Ministry of Industry and Information Technology, China

Xiaoyu Hou, School of Architecture, Harbin Institute of Technology; Key Laboratory of Cold Region Urban and Rural Human Settlement Environment Science and Technology, Ministry of Industry and Information Technology, China

Kudryavtsev Fedor, Moscow Institute of Architecture (state academy), Russia

Xiuqi Zhe, School of Art, Liaoning Finance \& Trade College, China

Pengluo Que, School of Architecture, Harbin Institute of Technology; Key Laboratory of Cold Region Urban and Rural Human Settlement Environment Science and Technology, Ministry of Industry and Information Technology, China

Xiaoyu Jia, China Urban Construction Design \& Research Institute,China

\begin{abstract}
With the comprehensive popularization of Internet of Things technology and mobile terminals, social media has completely changed the social lifestyle of human beings with a new type of communications. Cities, as the physical representation and spatial support system of social activities, are also fully digitalized and computerized. In urban cognition activities, social media has been proved by natural experiments is an important way to obtain information, whether in the preliminary planning and field space cognition stage, showing obvious coupling, and the strong coupling relationships of cognitive objects further confirms the importance of social media. At the same time, the traditional cognition model based on human instinct is the foundation and ultimate goal-seeking of media cognition, it is still the foundation of urban planning theory research and urban construction in the media age. As a new communication technology, social media is constantly promoting people's width and depth of people's urban environment cognition, and it is the basis for the comprehensive computational transformation of the urban planning discipline.
\end{abstract}

\section{Keywords}

Media Society, Urban Cognitive, Natural Experiment, Chongqing, China

\section{Introduction}

With the development of computer technology, especially the full popularity of Internet technology and mobile terminal, milestone changes have taken place in data production methods, data transmission methods, and data analysis modes, marking the arrival of the Era of Big Data. Every minute of every day, our planet now pulses with trillions of digital social signals, bombarding us with streams of status updates, news stories, tweets, pokes, posts, referrals, advertisements, notifications, shares, check-ins, and ratings 
for peers in our social networks, news media, advertisers, and the crowd. These signals are delivered to our always-on mobile devices through platforms like Facebook, Snapchat, Instagram, YouTube, Twitter, WeChat and TikTok, and they are touted through the human social network by algorithms. (Sinan Aral, 2000) The New Media Society, since which was invented, have promoted the development of social, working, shopping, and travel methods completely changed, fully digitized, algorithmized, and computationalized. Cities, as the physical representation and spatial container of human society, have been digitally transformed accompanying human society, that is, the digitization, algorithmization, computerization of urban form, urban planning theory. Under the background of the era of comprehensive computerization of urban planning disciplines, the systematic study of the coupling relationship between digital media space and urban physical space cognitive has important reference significance for urban planning theory research and urban planning practice in the Era of Big Data.

This study takes the coupling relationship between digital media space cognition and real urban space cognition in the Era of Big Data as the specific research objective, takes Chongqing, an important city in central and western China as the specific research object, adopts natural experimental methods, and firstly uses social media to formulate urban space cognition Plan; secondly, through field cognitive to form urban space cognition report, and finally analyzes the coupling relationship between media space cognition and real space cognition through a comparative analysis of data between the cognition plan and the cognition report, and discuss the influence model and mechanism of the social media on urban space cognition, to provide theoretical support for the computational transformation of urban planning discipline in Era of Big Data, so as to better respond the needs of the digital transformation of human society, and to realize the common ideal of "Time Well Spend" and "Better City, Better Life".

\section{Methodology}

\subsection{Urban Cognitive Experiment Design}

\subsubsection{Basic Condition of Cognitive Experiment}

The experiment is based on the "City Environment Cognition" course I opened for School of Architecture, Harbin Institute of Technology (abbreviated as HIT). Course nature: social practice class, the first professional cognition practice carried out at the end of first year basic knowledge study. Course object: urban planning and landscape architecture student. Course purpose: through the city cognitive practice, establish perceptual cognition of urban forms, urban planning discipline, and lay an intuitive foundation for subsequent professional learning. There are 62 students in this course. From the professional composition: 46 students majoring in urban planning, 16 students majoring in landscape architecture; from the student source: 4 students from Sichuan Province, 2 students from Chongqing (Chongqing is a municipality directly under the Central Government, not included in Sichuan Province), 46 students from other provinces; From the perspective of whether they have been to Chongqing: 17 people have visited Chongqing, 45 people have not visited Chongqing.

Does this experiment have representatives? The subjects of the experiment are the first year students majoring in urban planning. According to the urban planning major training program of School of Architecture, HIT, the first year student's professional training mainly focuses on the abstract understanding of space and the ability of hand-drawing expression, and the basic theories and basic skills to carry out urban space cognition. At the same time, the first year students basically did not receive the professional training of urban planning theory and planning methods, and basically do not preconceived professional knowledge impact, or are very small, which can be taken as enthusiast of urban space exploration. Therefore, taking the first year students of urban planning majors as experimental subjects, which are universal and representative. 


\subsubsection{Natural Experiment Method}

The Natural experiment is a design-based research method, which has become ubiquitous in the social sciences (Thad Dunning,2012). It is useful in the investigation of questions for which random assignment is either impracticable, uncertainty or unethical (Sinan Aral, 2000). The urban cognition, as a special type of the social experiment, adopting natural experiment is very scientific: First, the uncertainty of the cognitive subject: human. Human, the subject of urban cognition is a highly socialize existence. Although human cognition of the urban remains relative stable in a certain period of time, with the carry out of the cognition activities and the continue exchange of cognition information, the image of the city maybe be affected, maybe occur quantitative change and even essence changes. Second, the complexity of the urban system. The city is a complex giant system, and its information volume is supersaturated compared to human cognitive capability. In the stage of cognitive planning and specific cognitive practicing, there are constant stimuli of various information, and often change the established cognitive plan and induce new cognitive activities. Third, the unequal chance of random grouping. The urban environment cognition course is a very import practical segment in the teaching plan of the urban planning major of School of Architecture, HIT, and is of great significance to the student's follow-up professional learning. If adopting the random grouping method, although every student has equal opportunities to participate in or not participate in cognitive practice, but it is unfair and unethical for students who cannot participate in, because the future professional growth of students cannot be assumed. Therefore, the use of natural experimental methods in urban environment cognition research can not only overcome the variability of human subjects and the complexity of urban systems, but also avoid the inequality of random grouping and the immorality of future intervention.

\subsection{Variables and Data}

This research bases the urban environment cognition course teaching plan, adopts the natural experiment method, collects student's data of urban cognition plan and urban cognition practice, and then analyzes the coupling of the two sets of data, specifically including two stages: using social media to make the urban cognition plan and the carry out filed urban cognition in Chongqing. The specific variables and data are as follows:

\subsubsection{Urban cognition data in planning stage}

According to the teaching purpose of the city environment cognition course and the research design, the pre-urban cognition plan requires each students to use social media to select the 5 most wanted cognitive objects, and give specific ranking order, selection reasons, media paltform for information caquisition. the plann cognitive data is obtained in the form of questionnaires. In order not to induce or restrict student's selection, all survey information is filled in the blanks form to reflect students' cognitive wishes as true as possible. The specific results are as follows:

\subsubsection{Media platform data of cognition plan}

In the early planning stage, the main channels for acquiring urban cognitive information are social media platforms and real-world interpersonal communication. (Fig.1: information acquisition frequency stacking chart) First, the kinds of social media platforms. In the cognition planning stage, the social media used mainly include Baidu, Zhihu, Little Red Book, Ctrip, TikTok, BiliBili, Weibo, Gooood etc.. At the same time, the frequency of network or APP without specifying the media name is very high, as high as 106 times and 32 times respectively. Second, the kinds of social interpersonal communication information. Interpersonal communication methods or traditional information acquisition methods mainly include friend recommendation, professional books, ever visited, locals, etc., especially word-of-mouth opinions of friends, with a frequency up to 60 times. 


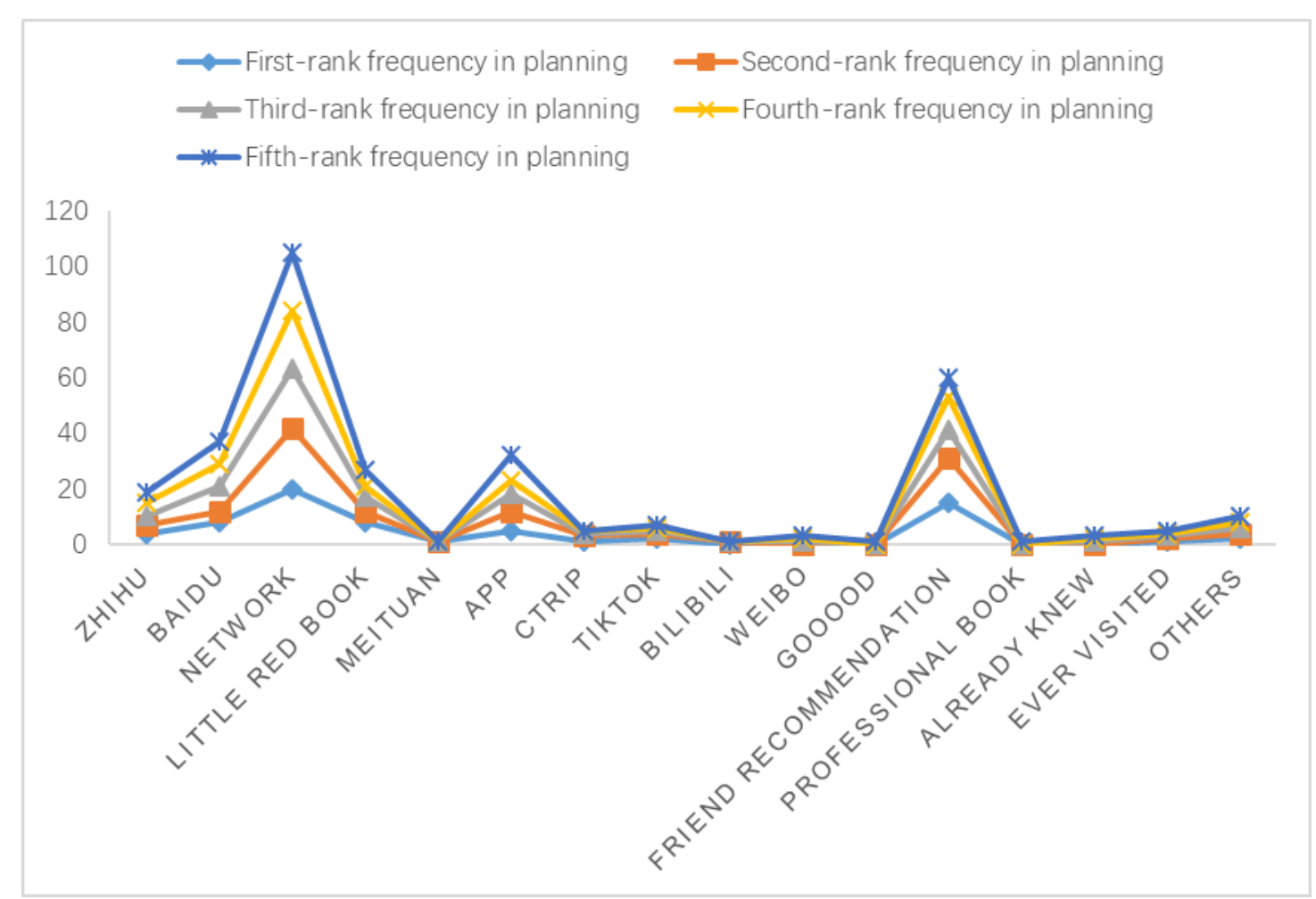

Figure 1. Accumulation diagram of media platform usage frequency in the planning stage. Source: Author.

\subsubsection{2. urban cognitive object data of plan}

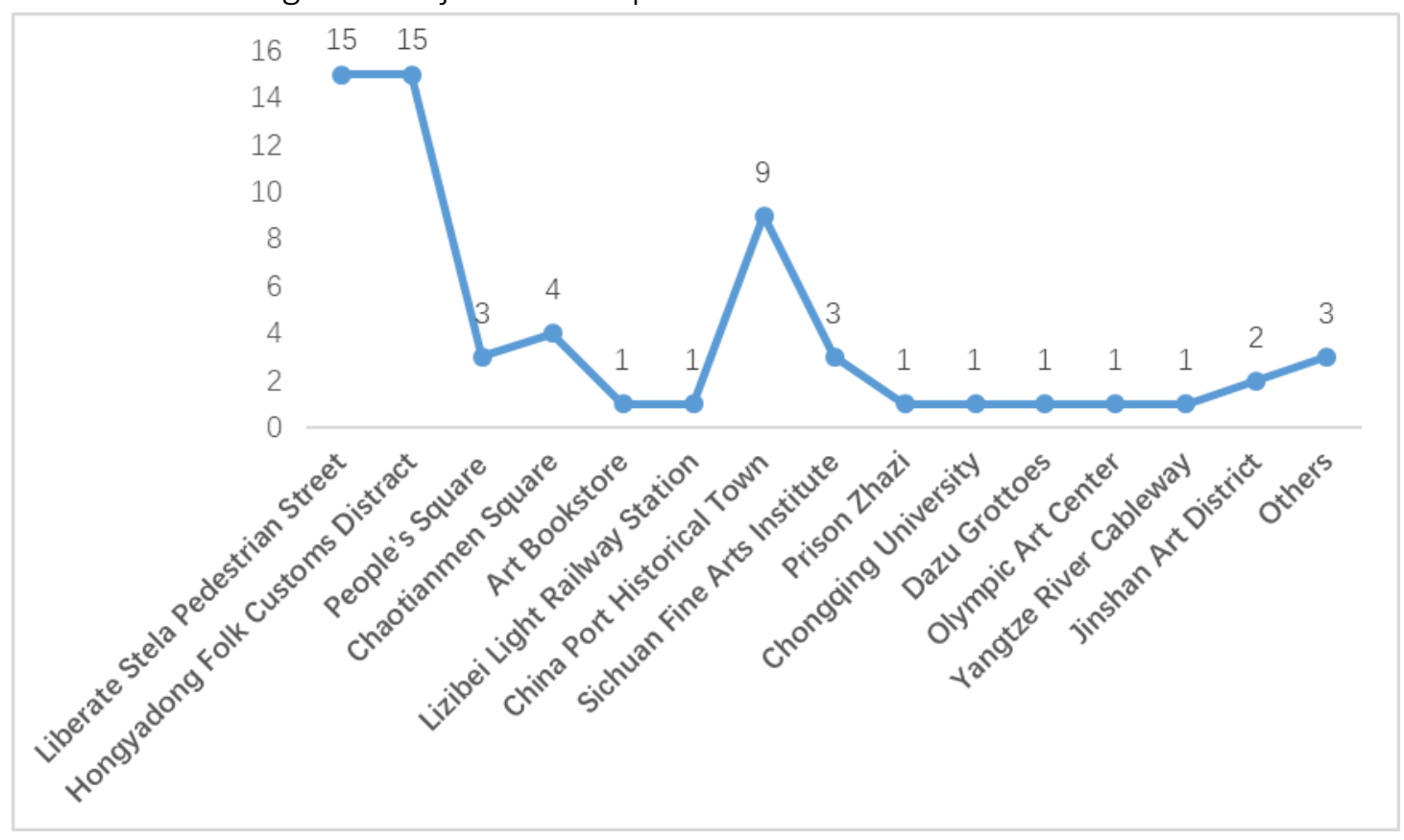

Figure 2. First rank cognitive object in the planning stage. Source: Author.

In the urban cognition planning stage, the survey of cognition object data also uses the form of filling in the blanks, requiring students to list the five cognition objects they most hope to investigate and the selection reasons. According to statistics, up to 65 cognitive objects were selected (Fig.2: First rank cognitive object in the planning stage), almost covering all aspects of Chongqing's urban characteristics. For example, the representative of Chongqing's modern construction, Chongqing People's Square, Chaotianmen Square, Olympic Art Center; the representative of Chongqing's mountain city 
characteristics, Hongyadong Folk Customs Distract, Mountain City Alley, Lizibei Light Rail Station, and Yangtze River Cableway; the Representative of Chongqing's history and culture, China Port historical Town, Liberate Stela Pedestrian Street, Hunan-Guangzhou Commercial Hall, Eling No.2 Factory Industrial Relics; the representative of Chongqing's modern red revolutionary culture, Mei Garden, Prison Zhazi, White Residence modern revolutionary historical sites; There are also Chongqing cuisine, such as, Bayi Snack Street, Choqing Hot Pot, and Chongqing Spicy Chicken. Among them, from the view of overall statistical frequency, the top ten cognition objects are: Hongyadong Folk Customs Distract, Liberate Stela Pedestrian Street, China Port historical Town, Chaotianmen Square, Lizibei Light Rail Station, Prison Zhazi modern revolutionary historical sites, People's Square, Yangtze River Cableway, Hunan-Guangzhou Commercial Hall.

\subsubsection{Urban cognition data in reporting stage}

According to the teaching purpose of the city environment cognition course and the research design, the field cognition report also requires students to select the 5 most impressive field cognitive objects, and give specific ranking order, reasons for selection, and information acquisition methods. The specific data is as follows:

\subsubsection{Media platform data of field cognition}

In the urban cognition reporting stage, compared with the previous cognition plan stage, in addition to relying heavily on social media planforms, the proportion of traditional urban cognition information acquisition methods has increased significantly. (Fig.3: Accumulation diagram of media usage frequency in the reporting stage) Specifically: First, the data of social media platform. The overall situation is similar to the planning stage. The main social media platforms used still are Baidu, Zhihu, Little Red Book, TikTok, Weibo, Gaode Maps, etc. the ratios of network and APP, no specific indicated, are still very high, the frequency of network is 98 times. Second, the data of traditional information acquisition methods. In the field urban cognition, traditional information acquisition methods, such as gradually discover during field cognition, word-of-mouth opinions of friends, attractions brochure, and locals recommendation, have been massively used, the frequency of gradually discover during field cognition was up to 86 times.

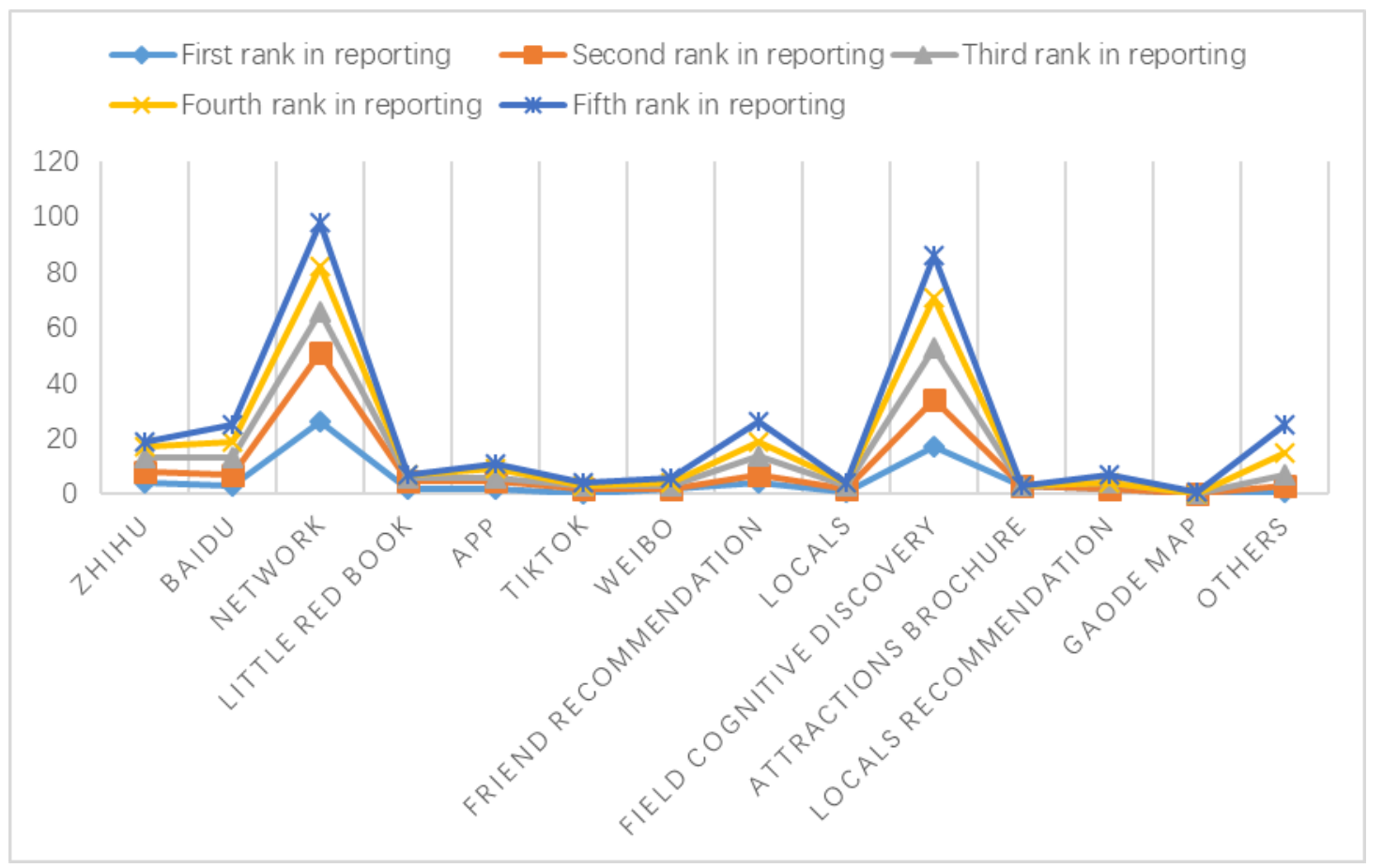

Figure 3. Accumulation diagram of media usage frequency in the reporting stage. Source: Author. 
2.2.2.2. Cognitive objects Data of field cognition

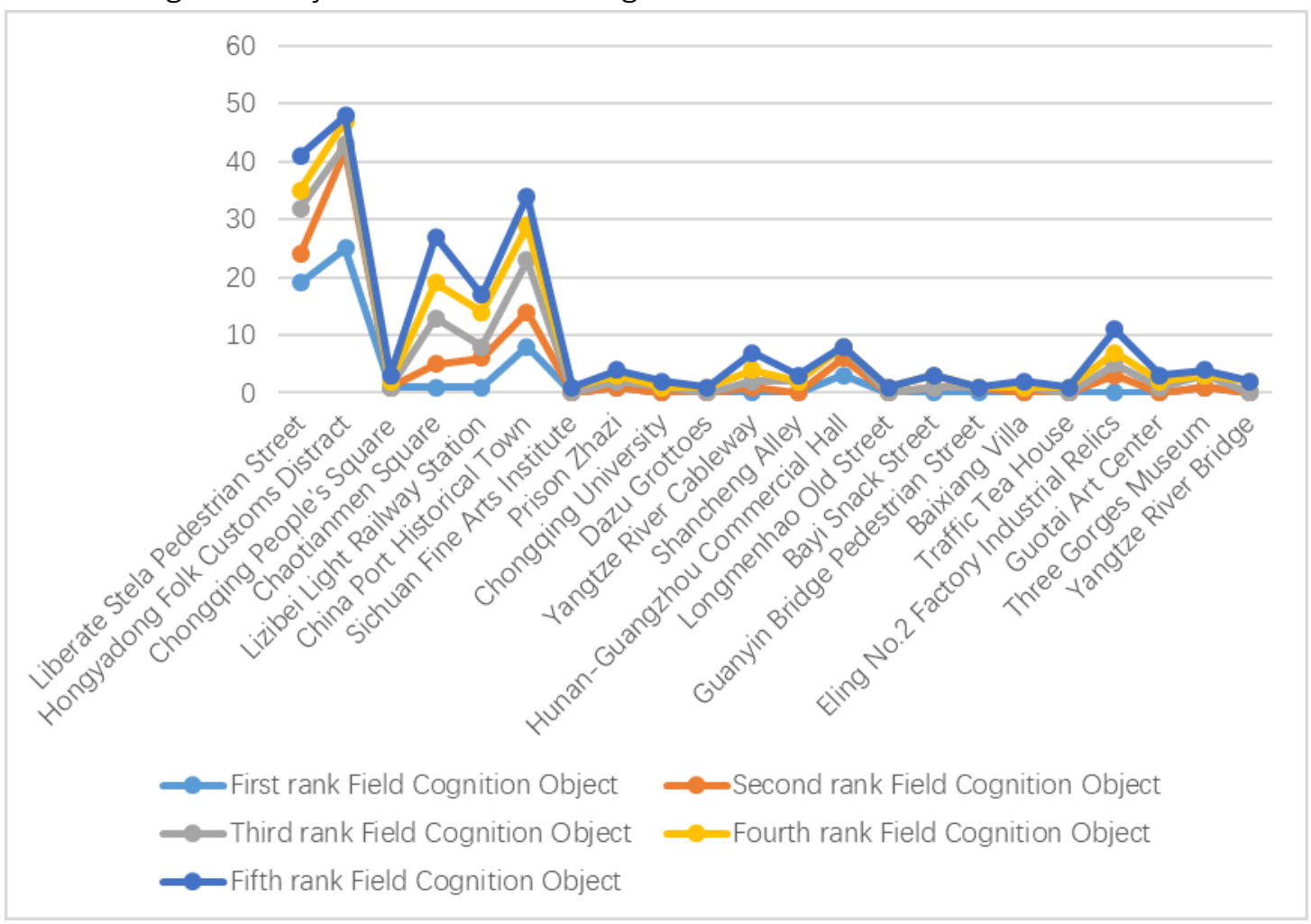

Figure 4-a. the line stacked chart of the summary of the top five field cognitive objects. Source: Author.

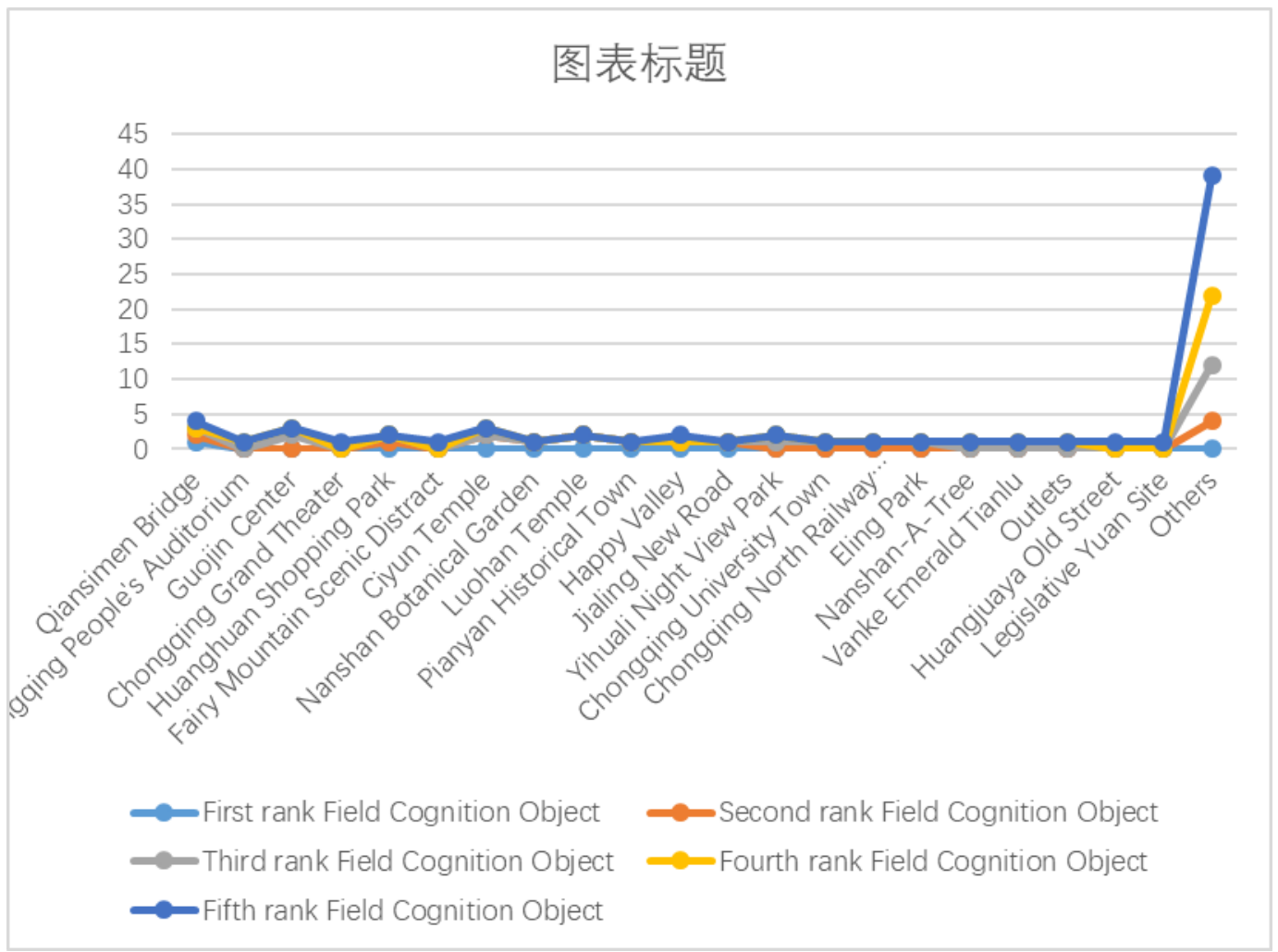

Figure 4-b. the line stacked chart of the summary of the top five field cognitive objects. Source: Author.

In the cognition reporting stage, through the student's field cognition practice, the top five urban cognitive object statistics are shown in Fig.4-a and Fig.4-b. there are a total of 44 cognitive objects, which 
is 21 fewer than the total number in the cognition planning stage, but the overall ranking the frequency of the top ten cognitive objects remained basically unchanged. For example, Hongyadong Folk Customs Distract is 48 times, Liberate Stela Pedestrian Street is 41 times, China Port historical Town is 34 times, Chaotianmen Square is 27 times, Lizibei Light Rail Station is 17 times, Eling No.2 Factory Industrial Relics is 11 times, Hunan-Guangzhou Commercial Hall is 8 times, Yangtze River Cableway is 7 times, Qiansimen Bridge is 4 times, Prison Zhazi modern revolutionary historical site is 4 times. In addition, the other category is up to 39 times, the specific content includes the keywords such as mountainous city, mountainous building, Yangtze River scenery, residential areas, street landscape and others unnamed urban cognition objects. The results echoed with the gradually discover during field cognition, reflected the spontaneity, systematic and comprehensiveness of the urban cognition.

\section{Coupling Analysis}

This research adopts the natural experiment to simulate the cognition process of human beings on the target city in the Social Media Era. That is, in the early planning stage, through social media retrieving the urban cognitive information, selecting the cognitive objects, forming the psychological expectations of the cognitive objects. In the later urban cognition practice stage, in the actual urban environment, through the multi-channel supplement of cognition information, the cognition objects will be continuously adjusted, finally forming the overall evaluation of the urban's cognition. Based on this, this research focuses on analyzing the coupling relationship of cognitive data of the planning stage and field cognition stage, specifically including the coupling relationship between the cognitive information acquisition platform data and the coupling relationship between the urban cognitive object data, and discussing the basic laws and basic patterns of the urban cognition.

\subsection{Coupling analysis of social media platform data}

\subsubsection{Coupling analysis of social media based on cognition object ranking}

From the above data sorting, Fig. 1 is the statistics of media platform usage based on the cognition objects ranking in the planning stage, and Fig. 2 is the statistics of media platform usage based on the cognition objects ranking in reporting stage. It can be seen that, regardless of the planning stage or the reporting stage, the methods of obtaining information in the rankings of cognitive objects are highly coupled, which shows that: First, the media loyalty of information acquisition. Media loyalty is the tendency of media choice. From the analysis of media platform usage frequency based on the cognition objects ranking, it can be seen that students' media selection is relatively consistent and stable throughout the entire process of the urban cognition, indicating that in the Era of Big Data, social media platforms have become the main information acquisition channels for people to carry out urban cognition, and show phased stability. Second, the social media dependence of information acquisition. From the analysis of information acquisition channels in the reporting stage, the frequency of the digital media usage is still very high, indicating that human beings are obviously dependent on digital media in current stage. At the same time, the frequency of use of traditional information acquisition methods is also very high, indicating that traditional information acquisition methods are the basis of human urban cognition and the only way for humans to achieve the ultimate cognition pleasure. Therefore, in urban cognition practice, the traditional information acquisition based on field cognition is the foundation and ultimate goal of cognition, but it also shows a clear dependence on social media.

\subsubsection{Coupling analysis of media platform between two stages}

Coupling analysis of media platforms in the planning stage and the reporting stage, using the media platform as the statistical dimension, respectively counts the number of uses among each ranking survey objects, and conducts the coupling analysis. In order to highlight the characteristics, this research uses the use frequency of social media in the information acquisition of the first-ranked cognitive object to 
conduct the two-stage coupling analysis, as shown in Fig.5: Line chart of the media usage frequency of the first-ranked cognitive. First, the coupling of the information acquisition mode between the planning stage and the reporting stage. In the two stages, the types and use frequency of social media are highly consistent, which once again proves that in the Era of Big Data, human's urban environment is highly dependent on social media, and it has become the basic way. Second, the expansion of traditional cognitive methods based on the media cognitive methods in field cognitive activities. Field urban cognition is a typical form of human perception of the living environment, a social interaction behavior that seeks the sense of security and identity, and the highly integrated information interaction behavior, such as gradually discovering during field cognition, attractions brochure, and local recommendation, etc. In particular, the cognition of non-iconic nodes, that are very important for shaping the characteristics of the city, is the immersive perception experience provided by the urban environment, and it is the information perception, information exchange and information expansion that must occur based on human cognitive instincts, is the inevitable result of the natural experiment of urban environment cognition. Therefore, although social media has become the most important way for us to obtain cognitive information, but in field cognitive activities, the information perception model based on human instinct still is the main information acquisition model, and the development of information technology only expands the ability of human beings to perceive the urban environment where we are live in.

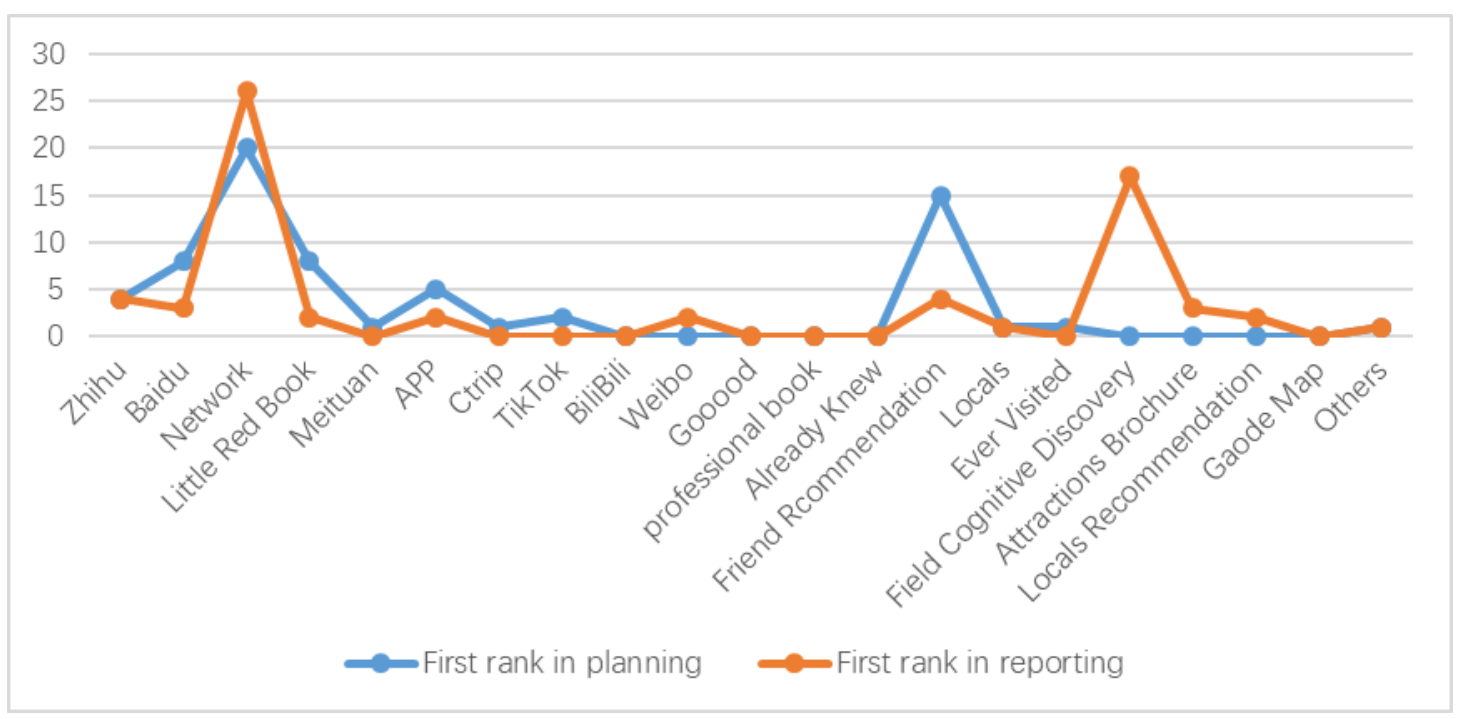

Figure 5. Line chart of the media usage frequency of the first-ranked cognitive. Source: Author.

\subsection{Coupling analysis of urban cognitive objects}

From the statistical data of cognitive objects in the planning stage and the reporting stage, the cognitive objects are very rich and basically cover all the typical characteristics of the mountain city Chongqing. According to the research design, the specific analysis as follows:

\subsubsection{Coupling analysis of cognition object among each ranking}

Performing statistical analysis of the ranking cognition objects in the planning stage and the reporting stage respectively, we can find: First, the concentration coupling of cognitive objects in each ranking. With the ranking order from first to fifth: On the one hand, the concentration of cognitive objects gradually weakens. Fox example, among the cognitive objects ranked first in the plan stage and in the report stage, Hongyadong Folk Customs Distract is 15 times in plan and 25 time in field, Liberate Stela Pedestrian Street is 15 times in plan and 19 times in field, China Port historical Town is 9 times in plan and 8 times in field. Correspondingly, among the cognitive objects ranked fifth in the plan stage and in the repot stage, Liberate Stela Pedestrian Street is 6 times in plan and 6 times in field, Eling No.2 Factory Industrial Relics is 5 times in plan and 4 times in field, Lizibei Light Rail Station is 4 times in plan and 3 
times in field, Yangtze River Cableway is 2 times in plan and 3 times in field. On the other hand, the coupling relationship between the two stages cognitive objects gradually weakened. In the plan stage and the report stage, there are a total of 27 cognitive objects ranked first, of which 17 have coupling relationships, and 10 have no coupling relationships; while there are a total of 39 cognitive objects ranked fifth, of which only 14 have coupling relationships, and 25 haven't coupling relationships. Second, the coupling analysis of the expansion degree of each ranking objects. Judging from the statistical data of each ranked cognitive object in the planning stage and the reporting stage, with the ranked order decreases, the concentration of cognitive objects gradually decreases in both. In comparison, the curvature of the reduction in the concentration of cognitive objects in the planning stage is greater, that is, the number of cognitive objects is greater with social media in the planning stage, seeing Fig.6: Histogram of Top-rank Cognition object in the planning and field stage. The reason is that the selection of cognitive objects through social media in the planning stage is break away from the actual urban environment and adopt the God perspective can browse whole information; In field cognation process, adopting ant perspective, and affected by the saturation impact of urban information, and more easily disperse the energy, and pay more attention to the non-landmark, non-node urban space. At the same time, in the actual urban environment, it is easier to form the identity sense and continuously strengthen through the environment information that has been paid attention to, thereby continuously narrowing the people's cognitive spectrum.

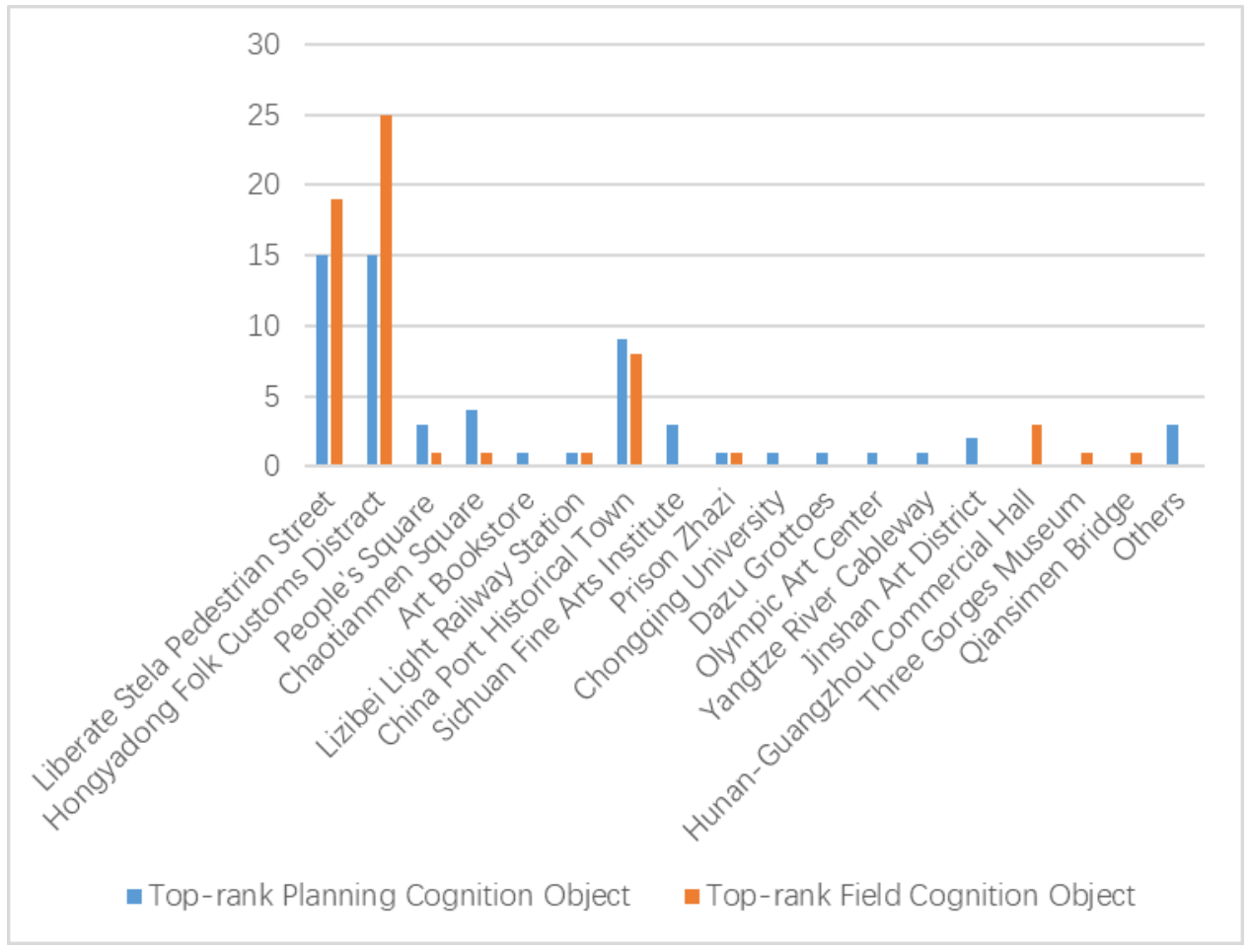

Figure 6. Histogram of Top-rank Cognition object in the planning and field stage. Source: Author.

\subsubsection{Coupling analysis of cognition object among each ranking}

Through the coupling analysis of the overall cognitive objects in the planning stage and the reporting stage, it reflects the role and mode of social media in the urban environment cognition at the macro level. First, the cognitive objects with strong coupling in the two stages in the first-ranked of planning and reporting cognitive objects, for example, Hongyadong Folk Customs Distract, Liberate Stela Pedestrian Street, China Port historical Town, Chaotianmen Square, Lizibei Light Rail Station, Yangtze River Cableway, Huan-Guangzhou Commerce Hall, Prison Zhazi modern revolutionary historical site, etc., show the urban cognition with the help of social media and the urban cognition in field are highly coupled in the category of urban landmarks. Second, the cognitive objects in the two stages have obvious long tail phenomena. In 
the planning stage, the first rank cognitive object contains 15 objects, the total number of cognitive objects in the second rank is 27 , in third rank is 37 , in fourth rank is 46 , in fifth is 56 . While, the reporting stage is similar to the planning stage. (Fig.7) Third, the overall coupling degree of the two-stage is relatively low. There are 70 cognitive objects included in two-stages, among which, 26 cognitive objects indluded in the planning stage were not actually implemented, at the same time, 16 in field cognitive objects were not included in the planning stage. The God's perspective of social media or the random discovery of field cognition both have the potential to meet the diverse needs of mankind. Meanwhile, it also shows that social media has very large limitations in the field of human's urban environment cognition at this stage, and the technical support behind it still needs considerable development, such as social media's simulation of human cognitive models, social media coding, response, and optimization and so on. Or to take it a step further, while social media is advancing social change, questions such as how to advance the digital and computational transformation of urban planning discipline are waiting for us to answer.

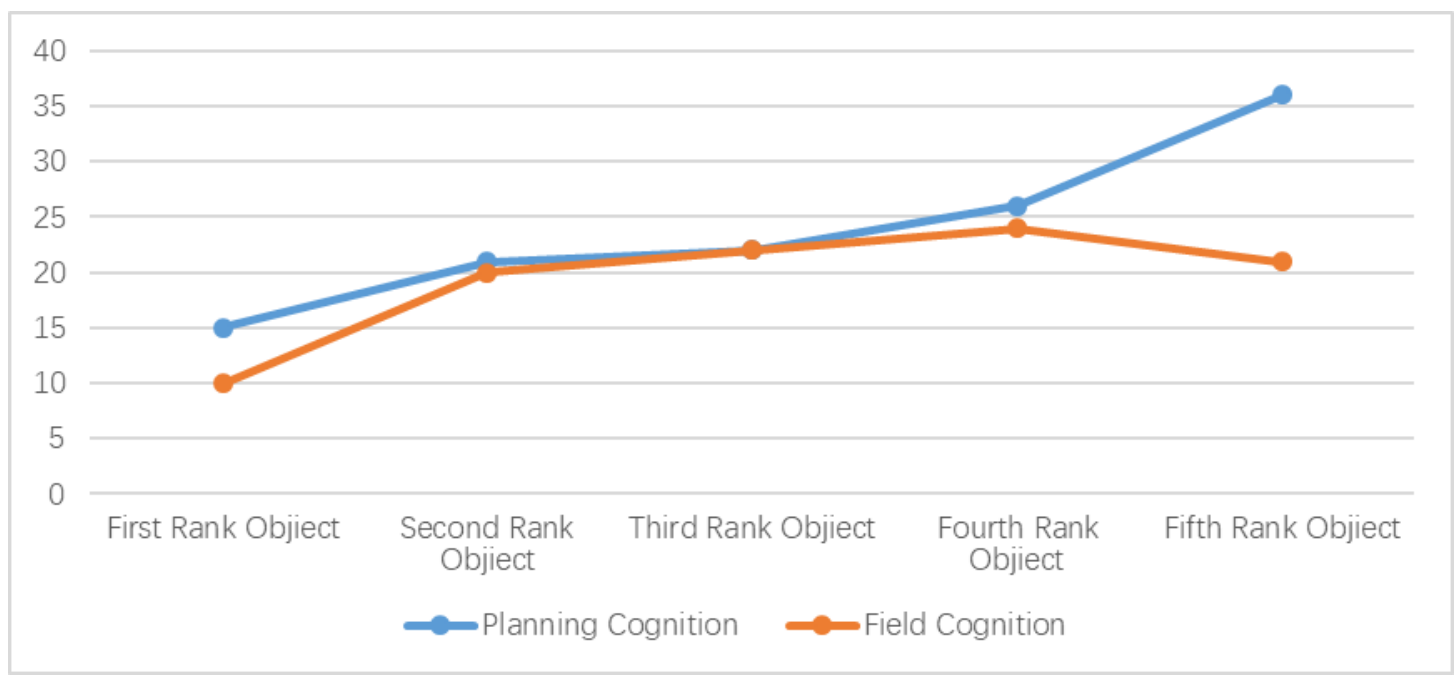

Figure 7. The number of cognitive object types among each rank. Source: Author.

\section{Conclusions and Discussion}

\subsection{Concluding Remarks}

Social media algorithms based on human physiology and social needs are continuously advancing the hypersocialization, large-scale personalized mass persuasion, and social polarization, which have triggered the full digitization and algorithm of human society, carrying out the coupling analysis of digital media space cognition and field actual urban space cognition is the basis for building the new urban science: computational city science.

\subsubsection{Coupling relationship of information acquisition between media cognition and field cognition}

First, media cognition and field cognition are highly coupled in the use of social media, regardless of the type and frequency, it indicates that social media has become the necessary information acquisition method for human urban's cognition. Second, the degree of specialization of social media used for media cognition and field cognition is not high. Generalized search with web and App, Baidu accounts for a very high proportion, while the use rate of professional websites such as Ctrip, Mafengwo, and Little Red Book are relatively not high, indicating that urban cognition has not yet become the focus area of social media industry. Third, the traditional human spatial perception model is still the basis of urban cognition in the media age. In field cognition, the information acquisition such as gradually discovering during field 
cognition, word-of-mouth opinions of friends, attractions brochure, and local recommendation still played an important role. From the research results, in the urban environment cognitive practice, the human spatial cognitive basic instinct is still the foundation, and social media has become an important assistant technology, whether it is in planning stage or in reporting stage, this can be regarded as human being explore process of the adaptive changes in the Big Data Era.

\subsubsection{Coupling relationship of cognitive objects between media cognition and field cognition}

First, the cognitive objects of media cognition and field cognition are highly couple in the curve head. Whether it is the cognitive plan made through social media or the cognitive report made through field cognition, it frequently captures the cognitive objects that best reflect the characteristics of Chongqing, indicating that the social media is highly effective in the cognition of the urban typical characteristics. Second, the cognitive objects of media cognition and field cognition are coupling in the long-tail of curve. When using social media to make cognitive plan, with the God perspective, the cognitive objects with lower rankings are obviously diffused; while in the field cognition, based on the characteristics of human spatial perception, it is affected by the saturation impact of urban environment information and the formation and strengthening of identification sense also have obvious tail diffusion. Although the two distribution are highly coupled, the causes of diffusion are completely different. Third, media cognition and field cognition are highly coupled with the overall form of cognitive objects, that is, both are long tail forms with peaks. In the peak part, there is both the coupling of cognitive objects and the coupling of cognitive frequency; while in the long tail part, there is only frequency coupling, and the specific cognitive objects vary greatly, which indicates the complexity of the urban giant system, and at the same time it also shows the difference between association analysis and causal analysis in urban research.

\subsection{Potential Contribution}

From the perspective of human development history, every change in human society, whether it is religion, politics, culture, or technology, fist occurred in the social field, and then transmitted to the field of urban construction, and solidified into the specific urban form, and it still existed a process of continuous adjustment and feedback over a long time. In the Era of Big Data, the digitization, algorithmization and computeralizaiton of the society, also will lead to the subsequent digitization, algorithmization and computeralization of the urban planning discipline. We are at the eve or beginning of the computeralization of the urban planning discipline, and we advocate the construction of the computational city science. This research is only a specific empirical study on the coupling of media space cognition and field space cognition, hoping to have certain stage benefits to explore how to use social media to recognize the urban, how to promote the spatial response and support to social digitization, and how to construct computational city planning theories and prototypes, how to guide computational city planning practice, etc., its value is, using the Chinese proverb, there is no way to reach a thousand miles without accumulating steps.

\subsection{Limitations and Next Steps}

Several limitations represent opportunities for further exploration. First, this study only explored the coupling relationship about the information acquisition methods and urban cognitive objects, other factors affecting cognition could be introduced in further studies. Second, this study uses natural experiment conjunction with Urban environment curriculum, the number of samples has certain limitations, follow-up studies will increase the samples number to make the research more scientific. Third, this study only uses the correlation analysis, the further study will carry out the causal analysis. Moreover, similar analyses should be undertaken in other megacities in China or other countries to provide a "big picture" and generate universal value. This research is part of the achievement of the 
Harbin Institute of Technology's Urban Environment Cognition Curriculum. We will incorporate relevant issues into the curriculum construction in 2022 to promote research in this field.

\section{References}

Zhang,E.J., Ye, Y., Hou, J.X. and Long, Y. (2021), Revealing the Spatial Preferences Embedded in Online Activities: A Case Study of Chengdu, China. In S.C.M. G., Christopher,P., Robert,G.,Aija.S. (Eds.). Urban Informatics and Future Cities [M]. Springer, 2021: 173-188.

Dunning, T.(2012). Natural Experiments in the Social Science: A Design-Based Approach. Cambridge University Press.

Sinan,A. (2020) The Hype Machine:How Socail Media Disrupts Our Elections, Our Economy, and Our Health-and How We Must Adapt[M]. New York: Currency.

Sadowski,J. (2020) Cyberspace and cityspace: On the emergence of platform urbanism. Urban Geogr. [online]. Available at:https://doi.org/10.1080/02723638.2020.1721055 (Accessed: 26 August 2021).

Salvia, G. and Morello, E. (2020) Sharing cities and citizens sharing: Perceptions and practices in milan. Cities 98. [online]. Available at:https://doi.org/10.1016/j.cities.2019.102592(Accessed: 18 August 2021).

Batty, M. (2018) Inventing future cities. The MIT Press, Cambridge.

Soroush,V., Deb,R., Sinan, A.(2018)The Spread of True and False News online, Science 350, np.6380:114651

Andrew, P.(2015) “Social Networking Usage: 2005-2015," Pew Research Center, [online]. Available at:http://www.pewinternet.org/2015/10/08/2015/Social-Networking-Usage-2005-2015/(Accessed: 18 August 2021).

Sanjeev, G. (2009) Connections: An Power of Our Social Networks and How They Shape Our Lives, Boston: Little, Brown Spark.

Herbert, A.S.(1971)“Designing Organizations for an Information-Rich World," in Martin, G.(ed.) Computers, Communication, and the Public Interest.Baltimore: Johns Hopkins University Press, pp: 40-41.

Casey,N.(2018) “Time Well Spent' Is Shaping Up to Be Tech's Next Big Debate," Verge, January 17

Laura, H.O.(2018)“Facebook Drastically Changes News Feed to Make It 'Good for People' (and Bad for Most Publishers)," NiemanLab, January 11, [online]. Available at: https://www.niemanlab.org/2018/01/facebook-drastically-changes-news-feed-to-make-it-good-forpeople-and-bad-for-mostpublishers/(Accessed: 20 August 2021).

Eli, P(2011)The Filter Bubble: What the Internet Is Hiding from You, London: Penguin UK.

Matthew, G. and Jesse, M.S.(2011)"Ideological Segregation Online and Offline," Quarterly Journal of Economics 126, no. 4 ,pp: 1799-839

Eytan, B., Solomon M. and Lada A.A.(2015) "Exposure to Ideologically Diverse News and Opinion on Facebook," Science 348, no. 6239, pp: 1130-32.

Jörg, C., Christian, P. And Ananya, S.(2019) "The Editor vs. the Algorithm: Economic Returns to Data and Externalities in Online News," November 12, [online]. Available at: https://papers.ssrn.com/sol3/papers.cfm?abstract_id=3479854(Accessed: 28 August 2021).

Benjamin, G. and Matthew, O.J.(2010)“Naive Learning in Social Networks and the Wisdom of Crowds,"American Economic Journal: Microeconomics 2, no. 1, pp: 114-15. 